\title{
Folate and epigenetics: why we should not forget bacterial biosynthesis
}

\author{
Dieuwertje E Kok ${ }^{*, 1}$, Wilma T Steegenga1 \& Jill A McKay² \\ ${ }^{1}$ Division of Human Nutrition and Health, Wageningen University and Research, Wageningen, The Netherlands \\ ${ }^{2}$ Department of Applied Sciences, Northumbria University, Newcastle upon Tyne, UK \\ *Author for correspondence: Tel.: +31 317485 901; dieuwertje.kok@wur.nl
}

First draft submitted: 20 July 2018; Accepted for publication: 27 July 2018; Published online:

20 September 2018

Keywords: biosynthesis $\bullet$ DNA methylation $\bullet$ epigenetics $\bullet$ folate $\bullet$ intestinal bacteria $\bullet$ microbiota $\bullet$ one-carbon metabolism

\section{Folate and epigenetics: why we should not forget bacterial biosynthesis}

Folate, together with other B-vitamins, plays a crucial role in epigenetic regulation. Folate-mediated one-carbon metabolism refers to a complex network of interconnected metabolic pathways, which ultimately result in a supply of methyl groups for DNA, RNA or protein methylation [1]. These epigenetic marks, particularly DNA methylation, have been proposed as plausible mechanisms underlying associations between folate and various disease outcomes, such as neural tube defects (NTDs), asthma, cardiometabolic disorders and cancer [2]. However, evidence for the impact of folate on most of these disease outcomes, except for NTDs, is hampered by inconsistencies [3], with studies investigating the relationship between folate, DNA methylation and disease being subject to further complexities (i.e., identification of specific genomic loci affected, directions of differential methylation and timing of epigenetic changes throughout the life course) [2].

Folate is closely related to other key role players in one-carbon metabolism (i.e., vitamin $\mathrm{B}_{12}$, homocysteine, methionine and choline) and therefore compensatory changes in inter-related metabolic pathways may account for inconsistent reports. Moreover, estimated folate intake does not necessarily correspond to circulating levels (correlations ranging from $r=0.05-0.54$ ) [4], pointing toward methodological issues or other genetic, physiological or environmental factors determining folate levels. One particular aspect that remains unexplored is the contribution of bacterial folate biosynthesis to folate status and DNA methylation patterns.

\section{Bacterial biosynthesis: an unexplored source of folate}

Mammalian cells are unable to produce folate, meaning it is an essential nutrient for which mammals are dependent on other sources. Folate is naturally present in several foods, including green leafy vegetables, fruits, cereals and liver products. Specific dietary supplements contain folic acid, which is a synthetic and stable form of folate. Many countries mandatorily fortify foods with folic acid to ensure sufficient levels in women of childbearing age and thereby reduce the risk of NTDs [5].

Besides these dietary sources of folate, there is convincing evidence that intestinal bacteria in the colon produce substantial amounts of folate as well as other B-vitamins [6]. Based on studies in piglets, who resemble humans in terms of folate absorption and omnivorous dietary habits, it is estimated that at least $18 \%$, and likely even more, of folate requirements can be achieved through bacterial folate biosynthesis [7]. While folate from dietary sources is predominantly absorbed in the small intestine, identification of folate transporters in the human colon implies that bacterially biosynthesized folate can be absorbed and participate in host metabolism [8]. These findings have been further substantiated with estimates that $\sim 322-396 \mu \mathrm{g}$ of folate per day can be absorbed in the human colon [9]. To put this into perspective, the average daily dietary requirement is $250-320 \mu \mathrm{g}$ of dietary folate equivalents for adults (European Food Safety Authority, 2014 and US Institute of Medicine, 1998). Interestingly, rodent studies suggest that bacterial folate biosynthesis can be stimulated through administration of prebiotic fructans or folate-producing bacteria resulting in increased serum or liver levels of folate [10]. It can therefore be speculated that the colon represents a local and substantial folate depot that participates in metabolism and contributes to circulating folate levels. The capacity of folate biosynthesis most likely depends on intestinal microbiota composition and function, 
and fluctuates within and between individuals, for example, as a consequence of dietary habits, use of antibiotics and other personal, lifestyle or clinical factors.

Although this field remains relatively unexplored, it is reasonable to assume that bacterial folate biosynthesis adds another layer of information with relevance for epigenetic marks. This concept provides interesting opportunities for further studies to investigate how bacterial folate biosynthesis may impact health throughout the life course. Two areas warranting particular attention, because of the recognized importance of folate and epigenetics in these respective areas, are early life development and colorectal carcinogenesis.

\section{Implications for early life development}

The importance of folate during early development is well established, particularly in the prevention of NTDs [5]. Although the role of folate in the multifactorial etiology of NTDs is not completely understood, mounting evidence points toward DNA methylation as mediating mechanism. Early life development, ranging from preconception to childhood, is considered a critical window characterized by rapid DNA methylation changes, pronounced susceptibility to environmental factors and programming of epigenetic marks that may have long-lasting health effects $[1,11,12]$. From this perspective, the intriguing question arises whether disturbance of maternal or infant colonic folate supply has consequences for one-carbon metabolism and epigenetic marks in this critical period. The composition of the infant intestinal microbiota changes rapidly in the early years, being influenced by many factors, including mode of birth delivery, feeding practice and antibiotic exposure [13]. Factors, in pregnancy or childhood, affecting establishment of the intestinal microbiota may therefore influence colonic folate depots. To our knowledge, no studies report associations between these factors and folate levels of mother or child, so far. However, maternal antibiotic use before or during pregnancy was associated with increased DNA methylation of an imprinted region in PLAGL1, and to a lesser extent IGF2 and MEG3 (for penicillin only) in cord blood [14]. Interestingly, methylation of these regions in cord blood was also inversely associated with maternal folate status within the same population [15]. This may imply that bacterial sources of folate impact DNA methylation, although underlying conditions may also contribute to the observed associations between maternal antibiotic use and offspring DNA methylation. Moreover, only a small panel of candidate imprinted genes or regions were considered, so far. DNA methylation of these genes or regions has been linked to other prenatal exposures, including calorie restriction during the Dutch famine or maternal anxiety, highlighting the overall plasticity of these regions $[16,17]$. Therefore, while these findings do not provide sufficient evidence for a definitive role of bacterial folate biosynthesis during early development, they provide a rational basis for further investigation.

Future studies with a genome-wide approach have the potential to complement the limited existing evidence. Studies with model species provide further opportunities to modulate bacterial folate biosynthesis and study the effects on developmental biology, for example by specific inhibition of the biosynthesis pathway (e.g., sulfonamide drugs) or use of probiotics containing folate-producing strains (e.g., specific lactic acid bacteria).

\section{Implications for colorectal carcinogenesis}

In adult life, folate status or intake has also been extensively studied in relation to epigenetic marks with potential relevance for health and disease. One of the organs that is of specific interest in relation to bacterial folate biosynthesis is the colon, given the proximity of the intestinal bacteria and the continuous exposure to locally produced folate. Colorectal cancer has been frequently studied in relation to folate intake or status [18]. Currently, it is thought that folate plays a dual role in colorectal cancer development, with either deficiency or excessive intake of folate or folic acid predisposing to an increased risk of colorectal cancer [18]. DNA methylation is proposed, together with DNA synthesis and DNA repair, as a plausible biological mechanism explaining these observations [18]. So far, the role of bacterial folate biosynthesis in colorectal carcinogenesis has not been studied in detail, although it has been postulated that bacterial metabolism may be a critical determinant of colorectal cancer risk [19]. O'Keefe and colleagues provided evidence that in native Africans, a population in which the colorectal cancer incidence is very low, the folate concentration in colonic content was $632 \pm 95 \mu \mathrm{g}$ [19]. In African-Americans, who are considered at relatively high risk of colorectal cancer, folate concentrations were comparable $(699 \pm 131 \mu \mathrm{g})$ to that of native Africans, despite higher dietary intake of folate ( $481 \pm 47$ and $201 \pm 23 \mu \mathrm{g} /$ day, respectively) [19]. This finding could be explained by differences in bacterial folate biosynthesis in the colon, although other factors, such as quality of dietary intake assessment and differences in bioavailability, might also be considered. While the contribution of bacterial folate biosynthesis in the context of DNA methylation needs to be explored in detail, an 
inverse association between colonic folate levels and DNA methylation of the tumor suppressor gene $M G M T$ in colonic tissue of 336 individuals without colorectal neoplasia substantiates the relevance of local folate depots [20].

In conclusion, although far from established, we propose that bacterial folate biosynthesis provides an additional source of folate with important implications for human health and disease. The constant supply of folate to the colon and subsequently to the circulation or other tissues may impact epigenetic marks at different stages throughout the life course. This concept aligns with the increasing awareness that bacterial and host metabolism are closely intertwined. Other examples of such interactions with relevance for epigenetics, include butyrate as a bacterial fermentation product and well-known histone deacetylase inhibitor. So far, bacterial folate biosynthesis remains relatively unexplored in the field of (nutritional) epigenetics. Interindividual differences in bacterial folate biosynthesis capacity may explain some of the inconsistencies reported between previous studies investigating the influence of folate on health outcomes and/or DNA methylation, especially those basing folate exposure assessment on dietary intake only. In this context, it is of specific interest to consider bacterial folate biosynthesis, in addition to dietary intake and circulating levels, in future studies.

\section{Acknowledgements}

The authors would like to thank Ellen Kampman for her mentorship, critical view and input in discussions.

\section{Financial \& competing interests disclosure}

DE Kok is supported by a Veni grant (016.Veni. 188.082) of The Netherlands Organisation for Scientific Research. The authors have no other relevant affiliations or financial involvement with any organization or entity with a financial interest in or financial conflict with the subject matter or materials discussed in the manuscript apart from those disclosed.

No writing assistance was utilized in the production of this manuscript.

\section{References}

1. McKay JA, Mathers JC. Diet induced epigenetic changes and their implications for health. Acta Physiol. 202(2), 103-118 (2011).

2. Crider KS, Yang TP, Berry RJ, Bailey LB. Folate and DNA methylation: a review of molecular mechanisms and the evidence for folate's role. Adv. Nutr.3(1), 21-38 (2012).

3. Bailey LB, Stover PJ, McNulty H et al. Biomarkers of nutrition for development - folate review. J. Nutr. 145(7), 1636S-1680S (2015).

4. Park JY, Vollset SE, Melse-Boonstra A, Chajes V, Ueland PM, Slimani N. Dietary intake and biological measurement of folate: a qualitative review of validation studies. Mol. Nutr. Food Res. 57(4), 562-581 (2013).

5. Honein MA, Paulozzi LJ, Mathews TJ, Erickson JD, Wong LY. Impact of folic acid fortification of the US food supply on the occurrence of neural tube defects. JAMA 285(23), 2981-2986 (2001).

6. LeBlanc JG, Milani C, de Giori GS, Sesma F, van Sinderen D, Ventura M. Bacteria as vitamin suppliers to their host: a gut microbiota perspective. Curr. Opin. Biotechnol. 24(2), 160-168 (2013).

7. Asrar FM, O’Connor DL. Bacterially synthesized folate and supplemental folic acid are absorbed across the large intestine of piglets. J. Nutr. Biochem. 16(10), 587-593 (2005).

8. Qiu A, Jansen M, Sakaris A et al. Identification of an intestinal folate transporter and the molecular basis for hereditary folate malabsorption. Cell 127(5), 917-928 (2006).

9. Lakoff A, Fazili Z, Aufreiter $S$ et al. Folate is absorbed across the human colon: evidence by using enteric-coated caplets containing 13C-labeled [6S]-5-formyltetrahydrofolate. Am. J. Clin. Nutr. 100(5), 1278-1286 (2014).

10. Pompei A, Cordisco L, Amaretti A et al. Administration of folate-producing bifidobacteria enhances folate status in Wistar rats. J. Nutr. 137(12), 2742-2746 (2007).

11. Smallwood SA, Kelsey G. De novo DNA methylation: a germ cell perspective. Trends Genet.28(1), 33-42 (2012).

12. Herceg Z, Ghantous A, Wild CP et al. Roadmap for investigating epigenome deregulation and environmental origins of cancer. Int. J. Cancer 142(5), 874-882 (2018).

13. Munyaka PM, Khafipour E, Ghia J-E. External influence of early childhood establishment of gut microbiota and subsequent health implications. Front. Pediatr. 2, 109 (2014).

14. Vidal AC, Murphy SK, Murtha AP et al. Associations between antibiotic exposure during pregnancy, birth weight and aberrant methylation at imprinted genes among offspring. Int. J. Obes. 37(7), 907-913 (2013).

15. Hoyo C, Daltveit AK, Iversen E et al. Erythrocyte folate concentrations, CpG methylation at genomically imprinted domains, and birth weight in a multiethnic newborn cohort. Epigenetics 9(8), 1120-1130 (2014).

16. Mansell T, Novakovic B, Meyer B et al. The effects of maternal anxiety during pregnancy on IGF2/H19 methylation in cord blood. Transl. Psychiatry 6, e765 (2016). 
17. Tobi EW, Slagboom PE, van Dongen J et al. Prenatal famine and genetic variation are independently and additively associated with DNA methylation at regulatory loci within IGF2/H19. PLoS ONE 7(5), e37933 (2012).

18. Williams EA. Folate, colorectal cancer and the involvement of DNA methylation. Proc. Nutr. Soc. 71(4), 592-597 (2012).

19. O'Keefe SJ, Ou J, Aufreiter S et al. Products of the colonic microbiota mediate the effects of diet on colon cancer risk. J. Nutr. 139(11), 2044-2048 (2009).

20. Hanks J, Ayed I, Kukreja N et al. The association between MTHFR 677C $>$ T genotype and folate status and genomic and gene-specific DNA methylation in the colon of individuals without colorectal neoplasia. Am. J. Clin. Nutr. 98(6), 1564-1574 (2013). 